\title{
Effectiveness of Local Hemostatic Agents in Epistaxis
}

\section{Tahir Hussain}

\section{ABSTRACT}

Background: Epistaxis is the commonest otorhinolaryngological emergency affecting up to $60 \%$ of the population in their lifetime with $6 \%$ requiring medical attention.

Objective: The aim of this study was to find out the epidemiological profile of epistaxis in our area and to find out the effectiveness of various available eight treatment options.

Materials and methods: This study was conducted to describe the etiological profile and treatment outcome of epistaxis. This was a prospective study of the cases of epistaxis from J uly 2012 to May 2013. Till date, the data regarding the management of epistaxis in our setting was not available. The purpose was to evaluate the clinical and management options for epistaxis in our settings.

Results: A total of 100 patients with epistaxis were studied. Males were affected twice more than the females (2.6:1). Their mean age was 48.63 years (4-82 years). The commonest cause of epistaxis was idiopathic (33\%) followed by trauma $(21 \%)$ and hypertension (18\%). Nonsurgical measures, such as light packing with cotton gauze soaked with local hemostatic (hemocoagulase) and antiseptic/antibiotic (36\%), silver nitrate cauterization $20(16 \%)$ and anterior nasal packing (15\%) and observation alone $(11 \%)$ were the main intervention methods in $78 \%$ of cases. Surgical measures mainly intranasal tumor resection was carried out in 3\% of cases.

Conclusion: The most common etiological factor for epistaxis is idiopathic. Most cases were successfully managed with conservative treatment alone and surgical intervention may not be necessary in most cases and should be the last resort.

Keywords: Epistaxis, Hemocoagulase, Idiopathic, Silver nitrate.

How to cite this article: Hussain T. Effectiveness of Local Hemostatic Agents in Epistaxis. Int J Head Neck Surg 2014;5(1):1-5.

\section{Source of support: $\mathrm{Nil}$}

Conflict of interest: None

\section{INTRODUCTION}

Epistaxis or nasal bleeding is recognized as one of the most common otorhinolaryngological emergencies worldwide and presents a challenge in resource-poor centers wherefacilities for caring of these patients are limited. E pistaxis is a problem frequently encountered in general practice and may present as an emergency, as a chronic problem of recurrent bleeds

\footnotetext{
Postgraduate Student

Department of ENT, Government Medical College, Srinagar J ammu \& Kashmir, India

Corresponding Author: Tahir Hussain, Postgraduate Student, Department of ENT, Government Medical College Srinagar, Jammu \& Kashmir, India, Phone: 9797737798 e-mail: thz47@yahoo.com
}

or may be a symptom of a generalized disorder. It affects the hemodynamic status as well as the psychological aspects of the patient and the accompanying attendants causing significant anxiety. Epistaxis is estimated to occur in $60 \%$ of persons worldwide during their lifetime, and approximately $6 \%$ of those with nose bleeds seek medical treatment. ${ }^{1}$ The prevalence increases for children less than 10 years of age and then rises again after the age of 40 years. ${ }^{1} \mathrm{G}$ enerally, males are affected more than females until the age of 50 , but after 50 no deference betw een sexes as reported. ${ }^{1,2,4,5,7}$ Epistaxis is commonly divided into anterior and posterior epistaxis, depending on the site of origin. ${ }^{1}$ A nterior nose bleeds arise from damage to Kiesselbach's plexus on the lower portion of the anterior nasal septum, known as the Little's area whereas posterior nose bleeds arise from damage to the posterior nasal septal artery. ${ }^{4,6} \mathrm{~A}$ nterior epistaxis is far more common than posterior epistaxis, accounting for more than $80 \%$ of cases. $^{4,6,7}$

The etiology of epistaxis can be broadly divided into the local or systemic causes, although even this distinction is difficult to make and the term 'idiopathic epistaxis' is ultimately used in about 80 to $90 \%$ of the cases. ${ }^{4,8}$ The etiological profile of epistaxis has been reported to vary with age and anatomical location. ${ }^{1-8}$ Traumatic epistaxis is more common in younger individuals (under age 35 years) and is most often due to digital trauma, facial injury, or a foreign body in the nasal cavity. ${ }^{1-8} \mathrm{~N}$ ontraumatic epistaxis is more characteristic of older patients (over age 50 years) and may be due to organ failure, neoplastic conditions, inflammation or environmental factors (temperature, humidity, altitude). ${ }^{1-8}$ Epistaxis that occurs in children younger than 10 years usually is mild and originates in the anterior nose, whereas epistaxis that occurs in individuals older than 50 years is more likely to be severe and to originate posteriorly. ${ }^{9}$ Epistaxis poses a greater risk in elderly people in whom clinical deterioration may progress rapidly, if the blood loss is significant. ${ }^{7}$

The treatment of epistaxis requires a systematic and methodical approach, and options vary according to the cause, location and severity of the hemorrhage. ${ }^{4,6,7,9}$ B oth conservative and surgical treatment modalities have been used in the treatment of epistaxis. ${ }^{2,6} \mathrm{H}$ owever, their outcome has never been evaluated in our setting partly because of paucity of local data. Most of the underlying causes of epistaxis are preventable. ${ }^{1-9} \mathrm{~A}$ clearer understanding of the 
causes, treatment and outcome of these patients is essential for establishment of preventive strategies as well as treatment guidelines. ${ }^{1,7,8}$ Such data is lacking in our settings as there is no local study which has been done on the subject. This study was conducted to identify the etiological profile and to determine the outcome of treatment of these patients. The results of this study will provide basis for planning of preventive strategies and establishment of treatment guidelines.

\section{MATERIALS AND METHODS}

This was a prospective study of patients who presented with epistaxis at SM HS Hospital, from December 2011 to December 2012.

The study subjects included all patients who presented with epistaxis at SM HS Hospital during the period under study. These patients were received either directly in Department of ENT or as referral from other departments. Initial assessment included hemodynamic status, type and severity of bleeding. If there were signs of excessive blood loss and/or patient was in a state of shock, steps were taken to stabilize the patient simultaneously with control of epistaxis. Resuscitation was carried out according to A dvanced Trauma Life Support (ATLS) principles. A fter resuscitation all patients underwent a detailed history taking and a through general examination, systemic examination and examination of the nose, throat and ears with special emphasis to identify the site of bleeding. The patients were subjected to investigations of hematological parameters and radiological evaluation. Blood samples were taken and sent for base line hemoglobin estimation and blood grouping and cross matching when indicated. Other rel evant investigations were ordered based on clinical suspicion regarding a particular etiology. The diagnosis of epistaxis was based on clinical history, physical findings, laboratory and radiological investigations with examination under anesthesia of the nose and nasopharynx; and biopsy was taken wherever necessary. A II patients were treated conservatively initially and surgical intervention was considered only when conservative means failed to control the epistaxis. Conservative (nonsurgical) treatment included light packing with gauze soaked with hemostatic agent and antisepticlantibiotic ointment, cauterization of the bleeding site using silver nitrate cauterization, el ectrocautery, anterior nasal packing and posterior nasal packing. Surgical treatment included resection of intranasal tumors. A rterial ligation and endovascular embolization were not performed as there were no patients with intractable epistaxis. Successful treatment was defined as no recurrent epistaxis following pack removal or no readmission with epistaxis within 24 hours of hospital discharge.

\section{Data Collection, Management and Statistical Analysis}

The data was collected using a pretested, structured proforma prepared for the purpose. Data collected included: patient's demographics, cause of epistaxis, anatomical location of bleeding sites, management modalities, need for blood transfusion, and length of hospital stay, complications and mortality. In descriptive analysis, the mean and standard deviation of continuous variables and percentages of categorical variables were computed.

\section{ETHICAL CONSIDERATION}

Ethical approval to conduct the study was sought from the institutional ethic review committee before the commencement of the study.

\section{RESULTS}

During the period under study, a total of 100 patients were studied. Seventy-seven (77\%) patients presented directly in department of ENT and 23 (23\%) were referred from other departments and hospitals. There were 75 males $(75 \%)$ and 25 females (25\%) with a mal e to female ratio of 2.6:1. Their ages ranged between 5 and 71 years (mean 48.63 years). The modal age group was 41 to 50 years (Table 1). The commonest cause of epistaxis was idiopathic (33\%) followed by trauma (21\%) and hypertension (18\%) (Table 2).

Nonsurgical measures were the main intervention methods in $88 \%$ of cases. Of this, light packing with cotton gauze soaked with local hemostatic (hemocoagulase) and antiseptic/antibiotic, local silver nitrate cauterization, observation alone without active intervention to arrest bleeding and anterior nasal packing were most common nonsurgical measures accounting for 36 and 16, 11 and 15\% respectively. Surgical measures mainly tumor resection was carried out in 3\% of cases (Table 3). According to the bleeding site, 88 patients (88\%) had anterior nasal bleeding, 12 (12\%) had posterior bleeding. The right nasal cavity $58(58 \%)$ was more affected than the left 32 (32\%). B ilateral involvement

Table 1: Age distribution

\begin{tabular}{lll}
\hline Age groups (yrs) & No. of patients & Age (\%) \\
\hline $0-10$ & 5 & 5 \\
$11-20$ & 15 & 15 \\
$21-30$ & 10 & 10 \\
$31-40$ & 7 & 7 \\
$41-50$ & 34 & 34 \\
$51-60$ & 19 & 19 \\
$61-70$ & 7 & 7 \\
$71-80$ & 3 & 3 \\
\hline
\end{tabular}


was recorded in $10(10 \%)$ of cases (Table 4). Blood transfusion was required in five $(5 \%)$ of cases. The overall success rate of treatment was $92.0 \%$. Success rates for various treatment modalities are shown in (Table 5). Prophylactic broad spectrum antibiotics were prescribed in all patients who had nasal packing, local cauterization and those who underwent surgical resection of intranasal tumors. The majority of patients $55(55 \%)$ wereadmitted in theENT wards and the remaining $45(45 \%)$ were treated as outpatients. Three (3\%) patients among the inpatients had severe head injuries and were referred to neurosurgery. M ost of inpatients were discharged between 1 and 4 days after treatment. The majority of patients ( $90 \%)$ had good recovery.

\section{DISCUSSION}

In this review, epistaxis was found to be more prevalent in patients older than 40 years, which is in agreement of

Table 2: Causes of epistaxis

\begin{tabular}{lll}
\hline Causes of epistaxis & Frequency & Age (\%) \\
\hline Idiopathic & 33 & 33 \\
Trauma & 21 & 21 \\
Rhinitis (inflammatory) & 14 & 14 \\
Hypertension/atherosclerosis & 18 & 18 \\
Tumors & 3 & 3 \\
latrogenic & 2 & 2 \\
Foreign body & 4 & 4 \\
Blood dyscrasias (Glanzmann's & 2 & 2 \\
thrombasthenia/hemophilia A) & & \\
Congenital heart disease (TOF) & 1 & 1 \\
Pregnancy & 3 & 3 \\
\hline
\end{tabular}

Table 3: Treatment modalities

\begin{tabular}{lll}
\hline Treatment modalities & No. of patients & Age (\%) \\
\hline Observation & 11 & 11 \\
$\begin{array}{l}\text { Light packing with gauze } \\
\text { (antiseptic/antibiotic/local }\end{array}$ & 36 & 36 \\
hemostatic) & & \\
Local trichloroacetic acid & 16 & 16 \\
(TCA) cautery & 15 & 15 \\
Anterior nasal packing & 3 & 3 \\
Posterior nasal packing & 5 & 5 \\
Endoscopic cautery & 0 & 0 \\
Surgical intervention & 14 & 14 \\
Combined procedures & & \\
\hline
\end{tabular}

Table 4: Bleeding sites

\begin{tabular}{lll}
\hline Sites of bleeding & $\begin{array}{l}\text { No. of } \\
\text { patients }\end{array}$ & Age (\%) \\
\hline Septum (ant/post) $(40 / 16) \%$ & 56 & 56 \\
Lateral wall (inf. turbinate/middle & 12 & 12 \\
turbinate/middle meatus) (9/11/1)\% & & \\
Floor (ant/post) (28/4)\% & 32 & 32 \\
\hline
\end{tabular}

Varshney and Saxena ${ }^{1}$ who reported most of their patients to be older than 40 years which correlates with other reports, which showed that epistaxis is a geriatric problem and with Eziyi et al. ${ }^{5}$ In the present study, epistaxis was found to affect more males than females, with a male to female ratio of 2.6:1. This male preponderance has been documented in literature. Globally, there is a male preponderance in epistaxis except in the geriatric age group in some reports where no significant sex difference exists. The male preponderance in this study may be attributed to high incidence of traumatic epistaxis which tends to affect young males because of their frequent involvement in high-risk taking behavior. Young males are the most active in the population and so are more vulnerable to trauma from nose picking especially among children, fights, road traffic accident with maxillofacial injuries causing epistaxis.

The present study shows that the most common cause of epistaxis was idiopathic causes followed by trauma and hypertension, which is consistent with other studies in developing countries. ${ }^{1,3,7,8}$ Trauma being the second most common cause. ${ }^{6} \mathrm{~T}$ his trauma varied from minor injury such as digital trauma to varying degrees of nasal injury from road traffic injury. Hypertension being the third commonest cause in this report shows epistaxis as evidence of poor blood pressure control. This is in keeping with an earlier report from N igeria of some patients who had epistaxis when their hypertension was not controlled due to cessation of antihypertensive drug therapy. ${ }^{12}$ Varsney and Saxenal in India recorded hypertension as the second commonest cause of epistaxis after idiopathic causes while Chaiyasate et al in Thailand reported hypertension to be the commonest cause of epistaxis followed by idiopathic causes so does $\mathrm{M}$ gbor. ${ }^{7}$ The need for regular blood pressure check and compliance to antihypertensive medications must be emphasized.

The management of epistaxis is well summarized in an age-old dictum: resuscitate the patient, establish the bleeding site, stop the bleeding and treat the cause of epistaxis. Dealing with a patient with active severe epistaxis can be bloody. The authors recommend universal precautions for all health care personnel involved in the care of these patients, including face mask with shields, gowns, hair coverage and double-gloving. The key to controlling most epistaxis is to find the site of the bleeding and cauterizing with silver nitrate or bi polar diathermy. ${ }^{4}$ The goal of treatment include: hemostasis, short hospital stay, low complication and cost effectiveness of the method of therapy. Controversy exists concerning the treatment that will best accomplish these goals. Treatment modalities can be separated into two groups; nonsurgical/conservative and surgical/interventional approaches. N onsurgical approach has been reported to stop 
Table 5: Success rates for various treatment modalities

\begin{tabular}{llll}
\hline Treatment modality & Number of patients & $\begin{array}{l}\text { Number of patients } \\
\text { treated successfully }\end{array}$ & Success rate (\%) \\
\hline Light packing with hemostatic/antiseptic/antibiotic ointment & 36 & 28 & 77.77 \\
Anterior nasal packing & 15 & 13 & 86.66 \\
Posterior nasal packing & 3 & 3 & 100 \\
Local TCA cauterization & 16 & 10 & 62 \\
Surgical resection of bleeding nasal tumor & 0 & 0 & 0 \\
Endoscopic electrocautery & 5 & 5 & 100 \\
\hline
\end{tabular}

the bleeding in more than 80 to $90 \%$ of cases. ${ }^{3}$ Of this, light packing with cotton gauze soaked with local hemostatic and antiseptic/antibiotic ${ }^{13}$ was most commonly used method. This form of treatment has not been extensively reported as an effective treatment, although we found in our study that it should be the first line of treatment following nose blowing/pinching keeping in view the discomfort, pain, infective complications and synechiae associated with A N P and many patients refusing the procedure in view of excessive pain. The few patients that had posterior nasal packing were mainly patients with hypertension. Posterior nasal packing was performed using gauze or ball loon Fol ey catheters inserted in the nasopharynx via the nostrils and inflated with sterile water. A nterior nasal packing was used in $15 \%$ of patients and was successful in $86.66 \%$ of them, while posterior nasal packing was successful in $100 \%$ of the cases where itwas tried. U rvashi et al ${ }^{4}$ reported successful use of anterior nasal packing in $83.5 \%$ case while posterior nasal pack was successful in $95.6 \%$ of cases. $N$ asal packing has the advantage of easy placement and removal; there was no need for an anesthetist or theater space for that treatment. It is al so affordable to the patients. Complications of nasal packing include septal hematoma, sinusitis and syncope during insertion of nasal pack, pressure necrosis of the al ae nasi, toxic shock syndrome. Most of our patients did not suffered this due to adequate precautions, such as technique of insertion of the pack, use of antibiotics and nasal decongestant were administered as some of the adjunct treatment to forestall this. The authors recommend use of prophylactic systemic antibiotics and nasal packing with antibiotic soaked gauze to minimize this complication (seeTable 5).

Cautery of the bleeding site can be performed chemically, electrically. Cauterization with Iaser or chemical (silver nitrate) was not used in our study because of their high costs and lack of availability. Cauterization in the form of electrical cautery was carried out for a group of patients where the bleeding points could be identified during examination. Electrical cauterization was used successfully in $100 \%$ of cases. This figure was higher than that reported by U rvashi et al ${ }^{4}$ in India. Nemer and $\mathrm{M}$ ottassim ${ }^{3}$ in J ordan reported a success rate of $74.0 \%$ which is lower than that of ours.
We did not encounter any postcautery complications, such as septal perforation or cartilage exposure. Since, cauterization of the bleeding point entails a good success rate and no complications it should, therefore, be the preferred modality of treatment where ever the bleeding site can be visualized. Rigid nasal endoscopy as part of the initial assessment in patients with epistaxis, with direct visualization and control of the bleeding point has been shown to be effective in the majority of patients, reducing the need for nasal packing.

In this study, surgical treatment was done only in 3\% of patients who presented with bleeding intranasal tumor and it was successful in $100 \%$ of them. Similar finding was also reported in studies. ${ }^{6,7} \mathrm{~N}$ o surgical ligation of any vessel or endovascular embolization was carried out on any patient in this study. A rterial ligation and embolization of feeding vessels are the last resort for intractable epistaxis.

The rate of blood transfusion for epistaxis has been reported in literature to range between 6.92 and $15.1 \%$ which is greater than our blood transfusion rate in our study. This high rate of blood transfusion is probably due to severe acute blood loss from the trauma sustained. The use of antimicrobial prophylaxis in the presence of nasal packing for the treatment epistaxis remains controversial. ${ }^{10}$ M ost of literatures recommend that patients with high-risk nasal packing should be started on prophylactic antibiotics, due to an increased risk for sinusitis and toxic shock syndrome. Blood soaked pack and raw mucosal surface are good media for bacterial multiplication resulting in infection including sinusitis and sometimes toxic shock syndromes. ${ }^{11}$

The mean length of hospital stay in our stay was 2 days which is shorter than that reported by other authors. ${ }^{5,3}$ Patients who underwent local cauterization were found to have significant shorter LOS compared to those with anterior nasal packing. Those requiring posterior nasal packing remained in hospital for an average of 6 days which is higher compared to those with local cauterization or anterior nasal packing. From our observations of average hospital stay with different treatment modalities, we are able to infer that cauterization of the bleeding point reduces hospital stay as compared to anterior nasal packing. However, the difference was not significant comparing anterior nasal packing and 
posterior nasal packing. Availability of nasal endoscopes which offers both proper visualization and direct facility for endoscopic cauterization to the area that is not easily accessible may have been able to further reduce the hospital stay and the discomfort of postnasal packing.

\section{LIMITATIONS}

Currently used methods for the treatment of epistaxis, like anterior nasal packing and posterior nasal packing, are very painfull and a sourse of great discomfort to patients and are used very frequently even in cases of minor bleed. U se of conservative measures, local hemostatic agents and direct visualization of bleeder followed by cautery should be the treatment of choice in case of nose bleed.

\section{CONCLUSION}

M ost cases were successfully managed with conservative (nonsurgical) treatment alone, such as light packing with hemostatic/antiseptic/antibiotic ointment, nasal packing and local cauterization. N onsurgical treatment is still useful to arrest nasal bleeding and it is safe and cost-effective, and surgical intervention should be the last resort. Light packing with hemostatic/antiseptic/antibiotic ointment should be tried in all patients, especially where facilities of nasal endoscopy, silver nitrate cautery are not available on OPD basis. Hemostatic used, in our study, was hemocoagulase (B otroclot).

\section{ACKNOWLE DGMENT}

I would like to thank all medical, paramedical staffs and my colleagues in the ENT Department for their great help.

\section{REFERENCES}

1. Varshney S, Saxena RK K. E pistaxis: a retrospective clinical study. Indian J Otolaryngol Head Neck Surg 2005 A pril-J une; 57(2): 125-129.

2. Gilyoma J M, Chalya PL. Etiological profile and treatment outcome of epistaxis at a tertiary care hospital in N orth-Western Tanzania: a prospective review of 104 cases. B M C Ear, Nose and Throat D isorders 2011;11:8.

3. N emerA K, M otassim A R. Evaluation of conservative measures in the treatment of epistaxis. K hartoum M ed J 2008;1(1):15-17.

4. U rvashi R, Raizada RM , Chaturvedi V N. Efficacy of conservative treatment modalities used in epistaxis. Indian J Otolaryngol Head N eck Surg 2004;56(1):21-23.

5. A kinpelu OV, A musa Y B, Eziyi JA E, Nwawolo CC. A retrospective analysis of aetiology and management of epistaxis in a South-Western Nigerian Teaching Hospital. West Afr J M ed 2009;28:165-168.

6. Peter A, K wabla A G, Isshalqd DM . Incidence of epistaxis in a Tertiary H ospital in Ghana. Natural Sci R esear ISSN 2224-3186 (Paper) 2225-0921 (Online) 2012;2(3):30-33.

7. M gbor N C. Epistaxis in Enugu: a 9-year review. Nig J Otorhinolaryngol 2004;1(1):11-14.

8. Okafor BC. Epistaxis: a clinical study of 540 cases. Ear Nose Throat J $1984 \mathrm{M}$ ar;63(3):153-159.

9. A bdul-K areem HA. Efficacy of different treatment modalities used in epistaxis. Iraqi J Med Sci 2010;8(1):18-28.

10. Biswas D, Wilson H, M al R. U se of systemic prophylactic antibiotics with anterior nasal packing in England, UK. Clin Otolaryngol 2006;31:566-567.

11. Gupta A, A grawal SR, Sivarajan K, Gupta V. A microbiological study of anterior nasal packs in epistaxis. Indian J Otolaryngol Head N eck Surg 1999;15(1):42-46.

12. Iseh KR, M uhammad Z. Pattern of epistaxis in Sokoto, Nigeria: a review of 72 cases. A nn A frican M ed 2008;7:107-111.

13. Özmen S, Özmen ÖA I I local ointment or cauterization more effective in childhood recurrent epistaxis. IntJ Paedia Otorhinolaryngol 2012 J une; 76(6);783-786. 